\title{
A Multiexit Recirculating Optical Packet Buffer
}

\author{
Wim A. Vanderbauwhede, Member, IEEE, and Hector Novella
}

\begin{abstract}
We propose a new type of recirculating buffer, the multiexit buffer (MEB), for use in asynchronous optical packet switches with statistical multiplexing, operating at speeds of 40-100 Gb/s. We demonstrate that the use of this type of buffer dramatically reduces the packet loss for a given buffer depth, thus reducing the buffer depth requirements and the overall cost of the optical packet switching. Physical layer simulation results show that it is possible to build this type of buffer with currently available active components. A hybrid optoelectronic control system is proposed, which allows control of the MEB with a minimum number of active components.
\end{abstract}

Index Terms-Buffers, optical communication, optical delay lines, packet switching.

\section{INTRODUCTION}

\section{A. Buffering for Optical Packet Switching (OPS)}

$\mathbf{T}$ HE OBJECTIVE of the OPSnet project is the design and implementation of a fast high-capacity OPS node that can switch packets of variable length in an asynchronous fashion. The OPSnet optical packet switch uses optical buffering with statistical multiplexing for contention resolution. The optical buffer is a parallel per-packet recirculating buffer with an egress port-transparent output multiplexer [1].

This letter reports the design of a novel type of recirculating buffer called multiexit buffer (MEB). The letter is structured as follows: the first part introduces the optical design of the OPSnet buffer, and makes the case for the MEB. The second part describes the proposed implementation of such a buffer, proposing a hybrid optoelectronic control system, and presents simulation results to demonstrate the viability of the proposed solution.

\section{B. OPSnet Buffer Design}

The OPSnet optical buffer design (Fig. 1) is called a parallel recirculating packet buffer. Every packet is stored in an individual recirculating buffer. The packet buffers are connected in parallel. To switch packets to the buffer, an active optical demultiplexer is required. The outputs of the packet buffer are combined using a passive multiplexer. The buffer control ensures that, at any instant of time, only one packet leaves its buffer bound for its destination port. Simultaneous releases are allowed if different packets are forwarded to different ports.

The main benefit of this design, as opposed to a serial buffer which would contain a number of packets in a single recirculating loop, is that it maximizes the reinsertion probability of the packets. Packets in a serial buffer take much longer for a

Manuscript received January 5, 2005; revised March 17, 2005.

W. A. Vanderbauwhede is with the Department of Computing Science, University of Glasgow, Glasgow G12 8QQ, U.K. (e-mail: wim@ dcs.gla.ac.uk).

H. Novella is with C. Estrella Antares, Gandia (Valencia) ES-46700, Spain (e-mail: hectorem@gmail.com).

Digital Object Identifier 10.1109/LPT.2005.851043

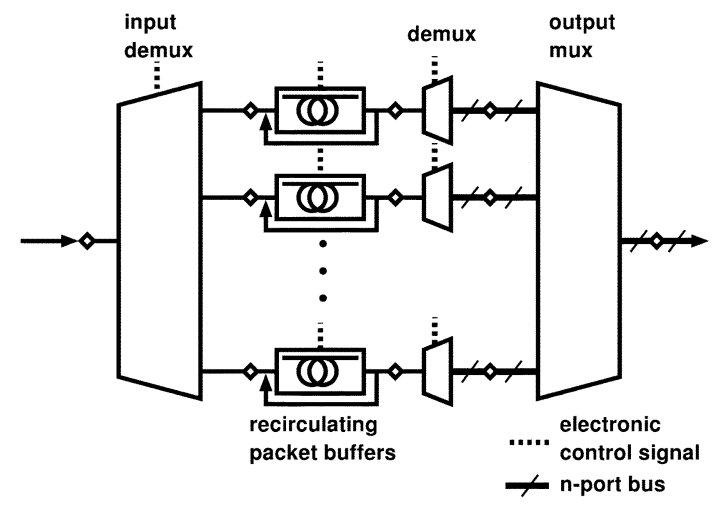

Fig. 1. Parallel circulating buffer design.

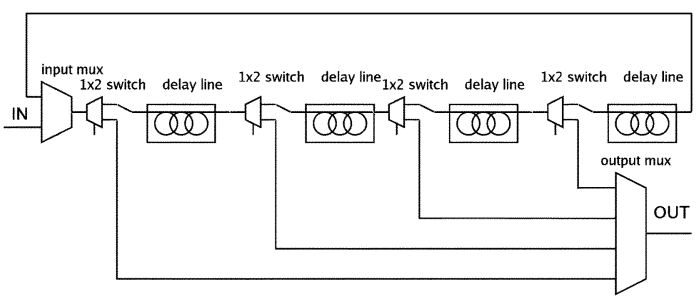

Fig. 2. Multiexit recirculating buffer.

round-trip. Furthermore, because every packet buffer is independent, this design allows for a much more sophisticated control, as the decision to shift the packet out of the buffer can be based on the packet order and packet priority.

\section{Multiexit Recirculating Buffer}

A recirculating buffer is essentially a closed-loop delay line with an input multiplexer and an output demultiplexer. The length of the delay line is determined by the maximum packet length. It is the closest optical equivalent of an electronic memory element: The optical packet circulates in the buffer loop until it can leave, just as an electronic packet would be stored in an electronic memory until it can leave. This type of buffer has extensively been reported [2]-[4]. The MEB design (Fig. 2) is a recirculating delay line with multiple exits at regular intervals along the loop. A passive demultiplexer combines the signals from all exits.

\section{Performance}

The egress probability for a packet in an MEB is proportional to the number of exits and inversely proportional to the loop delay. If the delay between subsequent exits is smaller than or equal to the duration of a gap in the traffic, then every gap will coincide with a packet egress request. In practice, this "ideal" number of exits is rather large (64 for Internet protocol (IP) over Ethernet at $100 \mathrm{~Gb} / \mathrm{s})$. However, as can be seen from Fig. 3, a much smaller number of exits (eight) already leads to a dramatic improvement in performance. For more than eight exits, increasing the number of exits has a relatively small impact. This 


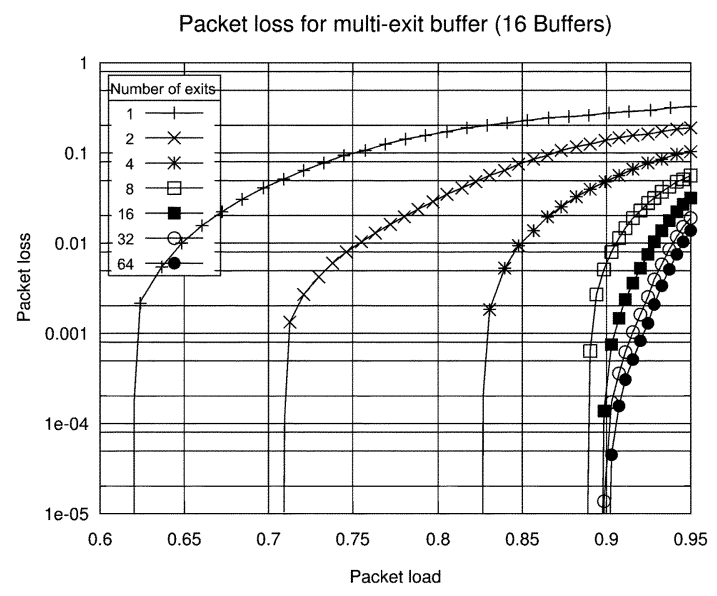

Fig. 3. Performance of the MEB for varying number of exits, compared to the single-exit buffer.

is a simulation of a four-port switch with a buffer depth of 16; the interarrival times have a negative exponential distribution.

From Fig. 3, it is very clear that the MEB requires a much smaller buffer depth than the single-exit buffer for the same load.

\section{E. Packet Sojourn Time}

Depending on the network load and the type of traffic, a packet will spend a longer or shorter time in the MEB. It is important to establish the longest sojourn time for a packet in the buffer, because this will impose the requirements on the physical layer components. Analysis of the distribution of the sojourn times for the same switch and traffic configuration as in Section I-D for an MEB with eight exits indicates that less than one packet in $10^{8}$ stay in the buffer for more than 40 loops; the number that stays for more than 30 loops is already very small (less than five in $10^{6}$ ). Consequently, the recirculating buffer can be designed to have an acceptable bit-error rate (BER) after recirculating no more than 40 times.

\section{MUltiEXIT BUfFER IMPLEMENTATION}

\section{A. Recirculating Buffer Implementation Issues}

As noted above, the MEB is conceptually simple: Instead of a single exit, there is a (possibly large) number of exits which are combined by means of a passive multiplexer, which is a logical OR (Fig. 2).

However, even for the simple fixed-length buffer with a single exit, the implementation is rather complicated. The main complicating factors are as follows.

1) The output demultiplexer has a finite switching time. This means that the signal to toggle the switch must arrive some time before the optical packet.

2) The signal in the recirculating buffer will deteriorate due to losses, noise, and distortion. So either the distortion and noise are kept to an acceptable level, or the signal must be regenerated; to compensate for the losses, an optical amplifier is required.

3) Due to the uncertainty on the effective index, it is very hard to know exactly the delay for a given length of the optical delay line. This means that it is not possible to synchronize the optical recirculating signal with an electronic clock.

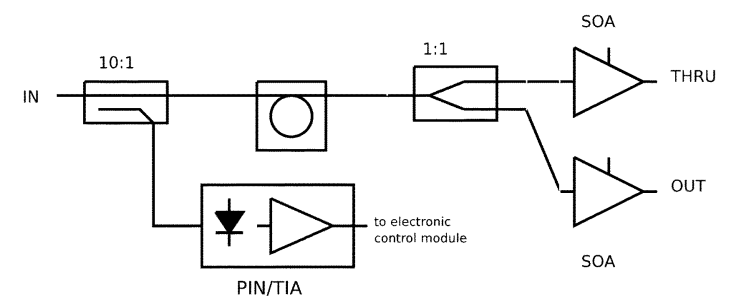

Fig. 4. Straightforward implementation of the MEB demultiplexing node.

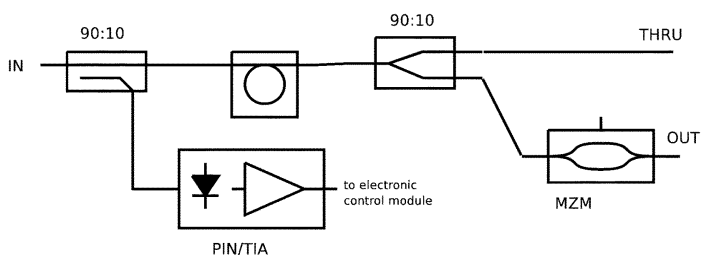

Fig. 5. Alternative implementation of the MEB demultiplexing node.

\section{B. Multiexit Buffer Design Issues}

A possible straightforward implementation of the MEB would be to build an output demux which detects the signal in advance, and switches all of the power (Fig. 4). It could be built with six components: two optical amplifiers [semiconductor optical amplifier (SOA)], a delay line, a 1:1 splitter, a 10:1 or higher ratio splitter, and a receiver (pin diode + transimpedance amplifier).

This approach has some obvious disadvantages.

- It requires a receiver and two optical amplifiers per exit, making it very expensive.

- Although SOAs amplify the signal, they also introduce distortion and noise. It would be very hard to regenerate a signal which travels through a series of SOAs, unless all SOAs would at least have $2 \mathrm{R}$ capabilities. But this would make the SOAs even more expensive.

An alternative could be to replace the SOAs by Mach-Zehnder modulators (MZMs) and keep a single gain-clamped SOA (GCSOA) to regenerate the signal [5]. The MZMs have two distinct advantages, as follows:

- they introduce no noise or distortion;

- they switch much faster than an SOA.

A further improvement, both in cost and losses, would be to eliminate the MZMs from the main loop, and only keep them in the exit branches (Fig. 5). The GC-SOA could be used to absorb the remaining signal, or a single MZM could be kept in the loop for this purpose.

As the MZMs are passive devices (i.e., no optical gain), the splitter should not be $1: 1$ but a smaller ratio (e.g., 10:1). The amount of power that can be tapped, and thus, the ratio and the number of exits, is determined by the lowest signal the GC-SOA can recover. Let $P_{\text {in }}$ be the input power, and $P_{\min }$ the lowest recoverable power level. This means the power at each tap as well as the power after traversing $N$ taps should not be lower than $P_{\min }$. Let $a_{N}$ be the attenuation due to waveguide loss per section. The splitting ratio at every tap can be calculated using $P_{i}=\left(P_{i-1}-P_{\min }\right) \cdot a_{N}$; the remaining power after $N$ taps is $P_{\text {min }}=P_{\text {in }} \cdot a_{N}^{N} / \sum_{i=0}^{N} a_{N}^{i}$. The latter equation allows a rough estimate of the acceptable waveguide loss. Assume $n=8$ (see Section I-D), $P_{\text {in }}=-7 \mathrm{dBm}$, and $P_{\min }=-25 \mathrm{dBm}$. For a 


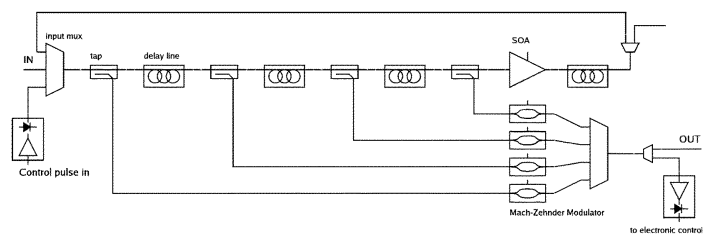

Fig. 6. Final proposed implementation of the MEB.

maximum-length Ethernet packet ( 1500 bytes) at $80 \mathrm{~Gb} / \mathrm{s}$, the buffer length is (\#bits/bitrate) $\cdot(c / n)=30 \mathrm{~m}$, and thus an estimate for the acceptable loss is $0.5 \mathrm{~dB} / \mathrm{m}$.

Finally, to reduce the number of receivers, which might be the most expensive components in the design, we propose following scheme (Fig. 6). The control system for this scheme is a hybrid optoelectronic solution.

- A control pulse is derived from the start of the packet. When a packet arrives at the OPS, the header is detected and processed. The optical and electronic paths will be synchronized by adjusting a tunable electronic delay line. The pulse must arrive at the exit switch control long enough in advance to allow for the switch delay and the electronic processing delays.

- This pulse is injected in the buffer loop at a wavelength different from that of the signal (this can even be an out-of-band wavelength). The pulse will travel through both branches of every subsequent tap. The MZM in its path will be transparent because it operates at a different wavelength.

- All derived pulses (the ones traveling in the exit branches) are combined with a star coupler and sent to a receiver.

- As a result, this receiver sees a pulse train and every pulse in the train will trigger a packet egress request. If there is no contention, the packet will be switched out. The original pulse (the one traveling in the loop) is dumped after the last tap.

- The arrival of the packet at the GC-SOA triggers a new pulse.

This solution reduces the number of receivers in the system from one per exit to one in total, but adds the cost of a transmitter. However, the transmitter requirements (spectral purity, wavelength accuracy, switching speed) are very relaxed, so the cost will be relatively low.

\section{Physical Layer Simulations}

To investigate the viability of the final proposal, physical layer simulations were done using a commercial software package (VPIsystems' VPItransmissionMaker). An MEB with eight exits was simulated. The crucial question is how long a packet can circulate in the MEB before the signal degrades to an unacceptable level. The signal degradation is determined by calculating the BER; a BER of $>10^{-12}$ is unacceptable.

The simulation setup was as follows.

- Transmitter: DFB laser with polarization controller (for simulation purposes only) and external MZM. Optical power $-7 \mathrm{dBm}$, modulation frequency $80 \mathrm{~Gb} / \mathrm{s}$, RZcoding; extinction ratio $10-20 \mathrm{~dB}$. Modulators with an extinction ratio of $13 \mathrm{~dB}$ at $40 \mathrm{~Gb} / \mathrm{s}$ are commercially available.

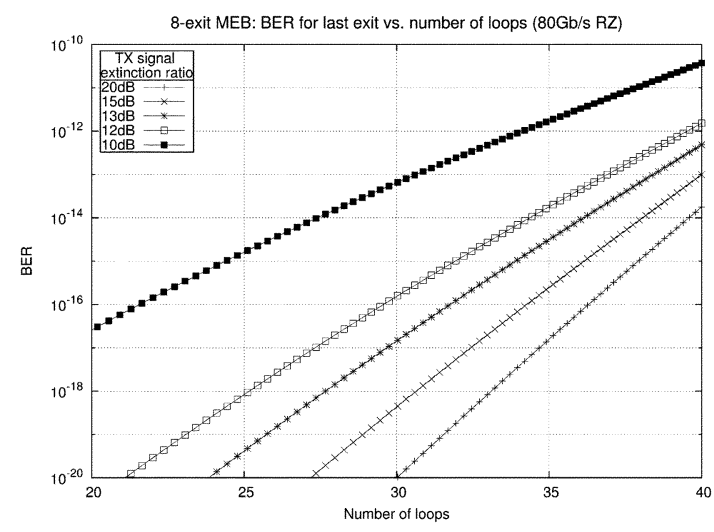

Fig. 7. BER for last exit (eight) versus number of loops for varying extinction ratio.

- Buffer: Every section consists of a variable-ratio (or absolute power) splitter, an MZM, and a delay line. We assume $\mathrm{SiO}_{x}$ waveguides with a loss of $0.5 \mathrm{~dB} / \mathrm{m}$; the buffer length is $30 \mathrm{~m}$ to accomodate a 1500-byte IP packet at $80 \mathrm{~Gb} / \mathrm{s}$.

- An SOA is used to reamplify the signal after the last exit. This is a simple SOA without automatic gain control or gain clamping, its length is $900 \mu \mathrm{m}$ and the drive current $90 \mathrm{~mA}$. This results only in amplitude regeneration, not pulse shaping.

- Receiver: PIN diode followed by a low-pass Bessel filter. The BER is taken for the last exit, because this is the most critical point. The results (Fig. 7) show that it is possible to build a multiexit recirculating buffer with a BER $<10^{-12}$ after 40 loops, with the current state of the art.

\section{CONCLUSION}

In this letter, a new design for a recirculating buffer for use in an asynchronous optical packet switch, called MEB, has been proposed. Simulation results show that this novel buffer design requires a much lower buffer depth than single-exit recirculating buffers.

An implementation which requires the lowest possible number of optical components and optical-electrical/ electrical-optical conversions has been proposed. The viability of this implementation has been verified using physical layer simulations.

\section{REFERENCES}

[1] W. Vanderbauwhede and D. Harle, "Novel design for an asynchronous optical packet switch," in Proc. 7th Conf. Optical Network Design and Modeling, Budapest, Hungary, Feb. 2003, pp. 737-754.

[2] K. J. Warbrick, P. R. Roorda, and D. Pugh, "Performance and scaling of a recirculating optical buffer," in Proc. London Communications Symp. 2000, London, U.K., Sep. 2000, pp. S10-2.

[3] Y. N. Singh and M. Naik, "Study of power variation of a buffered packet in optical loop buffer," in Proc. Photonics 2002, Mumbai, India, Dec. 2002, pp. NET-P11-NET-P11.

[4] A. A. Als, Z. Ghassemlooy, G. Swifth, P. Ball, and J. Chi, "Performance of the passive recirculating fiber loop buffer within an otdm transmission link," Opt. Commun., vol. 209, pp. 137-147, 2002.

[5] J.-T. Hsieh, S.-L. Lee, and J.-S. Wu, "All-optical 2R regenerator and decision gates by gain-clamped SOA s in a Mach-Zehnder configuration," in Proc. 8th Microoptics Conf., Osaka, Japan, 2001, Paper MOC01. 